\title{
Observation of Critical Phenomena and Self-Similarity in the Gravitational Collapse of Radiation Fluid
}

\author{
Charles R. Evans and Jason S. Coleman \\ Department of Physics and Astronomy \\ University of North Carolina, Chapel Hill, North Carolina 27599
}

(January 30, 2018)

\begin{abstract}
We observe critical phenomena in spherical collapse of radiation fluid. A sequence of spacetimes $\mathcal{S}[\eta]$ is numerically computed, containing models $(\eta \ll 1)$ that adiabatically disperse and models $(\eta \gg 1)$ that form a black hole. Near the critical point $\left(\eta_{c}\right)$, evolutions develop a self-similar region within which collapse is balanced by a strong, inward-moving rarefaction wave that holds $m(r) / r$ constant as a function of a self-similar coordinate $\xi$. The selfsimilar solution is known and we show near-critical evolutions asymptotically approaching it. A critical exponent $\beta \simeq 0.36$ is found for supercritical $\left(\eta>\eta_{c}\right)$ models.
\end{abstract}

PACS numbers: 04.20.Dw, 04.25.Dm, 04.40.Nr, 04.70.Bw 
New solutions of Einstein's equations at the threshold of black hole formation have recently been discovered. These solutions exhibit nonlinear dynamical behavior closely analogous to critical phenomena, and include power-law behavior, discrete scaling relations and a form of universality. All of these features were discovered in Choptuik's [1] studies of spherical collapse of massless scalar field. Abrahams and Evans [2] found strikingly similar behavior in axisymmetric collapse of gravitational waves, indicating the behavior is a generic feature of gravity. Confirmation has recently been made [3] of some of Choptuik's results. In this Letter, we report observation of critical phenomena in spherical collapse of radiation fluid and show the asymptotic approach of near-critical spacetimes to a self-similar solution near the center of collapse. This new result is fundamental in two ways; the self-similar solution has been separately and precisely calculated [4], and it exhibits local self-similarity.

The process for finding the threshold of black hole formation and critical phenomena in gravitational collapse has been described elsewhere [1,20,5,6]. Adopting a physical model with metric $\mathbf{g}$, matter fields $\phi_{A}$, stress-energy $\mathbf{T}\left(\phi_{A}\right)$, and symmetries, the future development is computed of elements of a family of Cauchy data, distinguished by a dimensionless strengthparameter $\eta$ and a length (or mass) scale $r_{0}$. Subcritical models $(\eta \ll 1)$ have smooth future development, dispersing all mass-energy to infinity, while a black hole appears in supercritical models $(\eta \gg 1)$ with only some mass-energy escaping. As $\eta$ is tuned in a search for the onset (at $\eta_{c}$ ) of black hole formation, black hole mass (for $\eta>\eta_{c}$ ) diminishes as a power law: $M_{\mathrm{BH}}=K\left(\eta-\eta_{c}\right)^{\beta}$, with $\beta>0$. In both scalar field collapse [1] and vacuum collapse [2,5], simulations give $\beta \simeq 0.37$, results that appear to be universal (i.e., independent of the parameterization of the initial data).

By implication, tuning the parameter to just the right value $\left(\eta=\eta_{c}\right)$ creates arbitrarily small black holes and regions of arbitrarily high spacetime curvature from smooth Cauchy data. Hence, it is argued [6] that the threshold of black hole formation is also the threshold of naked singularity formation. Spacetimes with $\eta=\eta_{c}$ are termed precisely critical.

In any physical model, many families of Cauchy data may exist with a critical point and a precisely-critical spacetime. Each family of data will have a characteristic proper length 
scale $r_{0}$. Related to this scale is the limiting interval, $\lim |T|=\left|T_{0}\right|$, as $\eta-\eta_{c} \rightarrow 0^{+}$, of proper time $T$ of the observer $\mathcal{O}$ centered in the collapse. Let $T=-T_{0}$ correspond to the initial data. Further, let $r$ be a proper spatial distance from $\mathcal{O}$. The central region of collapse $\mathcal{R}$ (in spacetime) exists for $r \ll r_{0},|T| \ll\left|T_{0}\right|$. Different precisely-critical spacetimes can be normalized, using scale invariance, to a common length scale $r_{0}$. These spacetimes will still differ on scales $T \sim-T_{0}$ and $r \sim r_{0}$ but approach a (physical-model-specific but otherwise unique) self-similar solution within $\mathcal{R}$. Within $\mathcal{R}$, all knowledge of the initial data is lost up to a single, dimensional constant.

Previous critical systems have displayed discrete self-similarities. Let $q$ denote dimension and let length have $q=1$. Allow the metric $\mathbf{g}$ to carry dimension $q=2$ [6]. For $\eta=\eta_{c}$, within $\mathcal{R}$ a map will exist under which $\mathbf{g} \rightarrow \mathbf{g}^{\prime}=e^{-2 \Delta} \mathbf{g}$, for a fixed value of $\Delta$. These are echoes resulting purely from nonlinearities. The scale factors $\Delta$ are pure numbers that emerge from the dynamics, with $e^{\Delta} \simeq 30$ in scalar wave collapse [1] and $e^{\Delta} \simeq 1.8$ in gravitational wave collapse [2, 2].

An analytic explanation of these phenomena would be important. This has been sought for scalar field collapse but the discrete self-similarity proved to be an impediment. The effort made obvious, though, that a local self-similarity would be more powerful, and suggested examining another system: perfect fluid collapse.

Imagine a spherical configuration of radiation fluid confined within areal radius $r_{0}$. Let the total gravitational mass be $M$. Define a dimensionless control parameter $\eta=2 M / r_{0}$ [8]. When $\eta \ll 1$, pressure dominates and should cause adiabatic expansion and dispersal of the fluid. When $\eta \gtrsim 1$, gravity should overwhelm pressure and spur the formation of a black hole. The onset of black hole formation should occur at some $\eta_{c} \sim 1$. One might anticipate, with a fluid, that any self-similarity emerging near $\eta_{c}$ will be local.

A successful attempt was made to find a self-similar solution representing the threshold of black hole formation in perfect fluid collapse [4]. Details of this solution will be given elsewhere; here we only describe the self-similar ansatz and a few numerical results. We assume a perfect fluid $T^{\mu \nu}=\rho U^{\mu} U^{\nu}+p g^{\mu \nu}$, with total energy density $\rho$, isotropic pressure 
$p$ and four-velocity $U^{\mu}$ satisfying $U_{\mu} U^{\mu}=-1$. A relativistic equation of state $p=(\gamma-1) \rho$ is adopted. In this paper, we specialize to radiation fluid $p=\frac{1}{3} \rho$. Adopting (dynamical) Schwarzschild coordinates, the line element is

$$
d s^{2}=-\alpha^{2} d t^{2}+a^{2} d r^{2}+r^{2} d \Omega^{2}
$$

with $\alpha(r, t)$ the lapse function and $a(r, t)$ the radial metric function. Constant proper sound speed $c_{s}=\sqrt{p / \rho}=\sqrt{\gamma-1}$ suggests the self-similar ansatz: $a(\xi), U^{r}(\xi), \rho(r, t)=$ $\Omega(\xi) / 4 \pi r^{2}, \alpha(r, t)=n r N(\xi) / t$, with self-similar coordinate $\xi=r / C(-t)^{n}$. Geometrically, $\mathbf{w}=(1 / n) t \partial / \partial t+r \partial / \partial r$ is the infinitesimal generator of the homothetic motion: $£_{\mathbf{w}} \mathbf{g}=2 \mathbf{g}$, $£_{\mathbf{w}} \mathbf{T}=0$. The similarity exponent $n$ is initially unknown, making the problem (in these coordinates) a type-2 self-similarity [9]. With this ansatz, the fluid and gravitational equations reduce to ordinary differential equations. These are solved by demanding regularity at $\xi=0$ $(r=0, t<0), \xi=\infty(r>0, t=0)$, and along the limiting, ingoing acoustic characteristic (sonic point) at $\xi=1$, across which the fluid might otherwise be discontinuous. The system is over-determined unless $n$ is taken as an eigenvalue of the nonlinear system. An analogous type-2 similarity problem is Guderley's shock implosion problem [10].

The similarity exponent was found to be $n \simeq 1.1485(\gamma=4 / 3)$. The solution is depicted in Fig. 1. A physical singularity exists at $r=0, t=0$, since $R_{\mu \nu} R^{\mu \nu}=\frac{256}{3} \pi^{2} \rho^{2} \sim T^{-4}$ and central proper time $T \sim(-t)^{n}$. Notice that $a$ does not approach unity as $\xi \rightarrow \infty$, but rather approaches $a \simeq 1.07$. Likewise, mass grows linearly with distance, with $m(r) / r \rightarrow 0.0596$ and $\Omega \rightarrow 9.56 \times 10^{-3}$, so the solution is not asymptotically flat. An asymptotically-flat spacetime would require truncating the self-similarity at some large $r$ [11]. Nonetheless, the self-similar solution is anticipated to represent the asymptotic behavior within $\mathcal{R}$ (i.e., as $r \rightarrow 0, t \rightarrow 0)$.

To confirm these expectations, we compute a series of models of spherically-symmetric collapse of radiation fluid, searching for a critical point and critical behavior. We adopt polar time slicing and radial gauge, retaining the form (1) of the line element, and assume a radiation fluid. We define $W \equiv \alpha U^{t}$ and $U \equiv a U^{r}$, with which the velocity normalization 
becomes $W^{2}=1+U^{2}$. Then, the equations of motion of the fluid, $\nabla_{\nu} T^{\mu \nu}=0$, are

$$
\begin{array}{r}
\frac{\partial}{\partial t}(a \rho W)+\frac{1}{r^{2}} \frac{\partial}{\partial r}\left(r^{2} \alpha \rho U\right)+p\left[\frac{\partial}{\partial t}(a W)+\frac{1}{r^{2}} \frac{\partial}{\partial r}\left(r^{2} \alpha U\right)\right]=0 \\
\frac{\partial}{\partial t}\left[a^{2}(\rho+p) W U\right]+\frac{1}{r^{2}} \frac{\partial}{\partial r}\left[r^{2} \alpha a(\rho+p) U^{2}\right] \\
+\alpha a(\rho+p)\left(W^{2} \frac{1}{\alpha} \frac{\partial \alpha}{\partial r}-U^{2} \frac{1}{a} \frac{\partial a}{\partial r}\right)+\alpha a \frac{\partial p}{\partial r}=0
\end{array}
$$

and the pressure is $p=\frac{1}{3} \rho$. In these coordinates, the gravitational field equations $G_{r}^{r}=$ $8 \pi T^{r}$ and $G^{t}{ }_{t}=8 \pi T^{t}{ }_{t}$ become

$$
\begin{aligned}
& \frac{1}{a} \frac{\partial a}{\partial r}+\frac{1}{2 r}\left(a^{2}-1\right)=4 \pi r a^{2} \rho\left[\gamma W^{2}-\gamma+1\right] \\
& \frac{1}{\alpha} \frac{\partial \alpha}{\partial r}-\frac{1}{2 r}\left(a^{2}-1\right)=4 \pi r a^{2} \rho\left[\gamma U^{2}+\gamma-1\right]
\end{aligned}
$$

with $\gamma=4 / 3$ adopted in the models we discuss here.

To construct a parameterized family of evolutions, we choose Cauchy data by specifying that the fluid be instantaneously at rest $V \equiv U / W=0$ and have an energy density profile

$$
\rho=\frac{1}{2} \pi^{-3 / 2} r_{0}^{-2} \eta \exp \left(-r^{2} / r_{0}^{2}\right)
$$

parameterized by $\eta$ and $r_{0}$. The total gravitational mass is $M=\frac{1}{2} \eta r_{0}$, so that $\eta=2 M / r_{0}$ is a dimensionless measure of the strength of the initial gravitational field, while $r_{0}$ is the typical initial length scale. Equations (4) and (5) are solved to complete the Cauchy data.

The dynamical behavior of these models is consistent with expectations. For $\gamma=4 / 3$ and this family of Cauchy data, the critical point is found in a bisecting search to be near $\eta_{c} \simeq$ 1.0188. For $\eta>\eta_{c}$, the impending formation of a black hole is observed. As is well-known, polar time slices avoid penetrating regions of trapped surfaces [12 and asymptotically approach the apparent horizon in collapsing spacetimes. When this occurs we have clear evidence of a black hole forming. Fig. 2 illustrates the form of the mass function 
$m(r)=\frac{1}{2} r\left(1-a^{-2}\right)$ late in a supercritical evolution. The "kink" where $r \simeq 2 m(r)$ locates the surface of the black hole and determines the hole's mass $M_{\mathrm{BH}}$. Immediately beyond the kink, $m(r)$ is nearly constant indicating an evacuated region that forms around the black hole. At larger radii, additional mass is evident, representing the fluid which did not collapse and is expanding outward.

Black-hole mass $M_{\mathrm{BH}}$ is found to be well described by a power law (see Fig. 3)

$$
M_{\mathrm{BH}} \simeq K\left(\eta-\eta_{c}\right)^{\beta}
$$

with a critical exponent $\beta \simeq 0.36$. This critical exponent value is numerically indistinguishable from those found in scalar wave collapse [1] and gravitational wave collapse [2], doubtlessly reflecting a deep property of the gravitational field equations.

The fluid is initially everywhere out of equilibrium. In near-critical models, the fluid in the inner region collapses while that in the outer region accelerates and expands outward. The collapsing central region is chased by a strong, ingoing rarefaction wave. As the radius $R(t)$ of the inner region diminishes, the rarefaction wave causes the mass to diminish also, keeping $m(R(t)) / R(t)$ nearly constant. The radius $R(t)$ of transition between collapse and expansion (edge of the rarefaction wave) decreases by several orders of magnitude in verynear-critical models. On fine scales, $r \ll r_{0}$, the fluid and gravitational field assume a self-similar form (see Fig. 4). Furthermore, the self-similar form being approached is the locally self-similar solution outlined above. As Fig. 4 shows, ultimately the self-similarity of the collapse is broken if $\eta \neq \eta_{c}$. Only a precisely-critical model would approach the selfsimilar solution and retain this dependence as $r \rightarrow 0, t \rightarrow 0$. Near- but not precisely-critical spacetimes develop a self-similar region on scales $r \ll r_{0}$ but then lose self-similarity on a scale determined by proximity in parameter space to the critical point: $r_{1} \simeq K\left|\eta-\eta_{c}\right|^{\beta}$. Self-similarity will only be apparent if $r_{1} \ll r_{0}$.

Approach to self-similarity within a region spanning two disparate scales is a familiar concept in hydrodynamics termed "intermediate asymptotics" [13]. The cardinal example is the modified Sedov-Taylor blast wave problem. Besides the usual Sedov-Taylor parameters 
of $E_{0}$, the blast wave energy, and $\rho_{0}$, the ambient gas density, the modified problem has a length scale $r_{0}$, within which the energy $E_{0}$ is arbitrarily distributed initially, and has a small pressure $p_{0}$ in the ambient gas. This small pressure introduces a second, new length scale: $r_{1}=\left(E_{0} / p_{0}\right)^{1 / 3} \gg r_{0}$. These two additional dimensional parameters break the self-similarity of the blast wave on length scales $r \lesssim r_{0}$ and $r \gtrsim r_{1}$ but on intermediate scales the solution asymptotically approaches the Sedov-Taylor self-similar form. There is a direct analogue in the behavior of near-critical spacetimes on scales between $r_{0}$ and $r_{1}$.

The self-similar solution determines the asymptotic properties of precisely-critical evolutions. Linear stability analysis should reveal the perturbative response of solutions nearby $\eta_{c}$ in parameter space and thereby perhaps provide an estimate of the critical exponent $\beta$. The self-similar solution, and therefore any precisely-critical evolution, apparently contains a naked singularity. However, the precisely-critical evolutions are likely to be a set of measure zero as there is extreme sensitivity to the initial conditions near $\eta_{c}$. Furthermore, the dependence of the lapse along $\xi=\infty(t=0)$ is $\alpha \sim r^{1-1 / n} \sim r^{0.129}$, indicating the singularity to be shrouded in infinite redshift.

C.R.E. acknowledges helpful conversations with A. Abrahams, M. Choptuik, R. Price and J. York. This research was supported by NSF grants PHY 90-01645, PHY 90-57865 and ASC 93-18152, which includes support from ARPA. C.R.E. thanks the Alfred P. Sloan Foundation for research support and thanks the Aspen Center for Physics for hospitality during summer 1993 where key aspects of this work were initiated. Computations were performed at the North Carolina Supercomputing Center. 


\section{REFERENCES}

[1] M. W. Choptuik, Phys. Rev. Lett. 70, 9 (1993).

[2] A. M. Abrahams and C. R. Evans, Phys. Rev. Lett. 70, 2980 (1993).

[3] C. Gundlach, R. H. Price and J. Pullin, submitted to Phys. Rev. D (1993).

[4] C. R. Evans, to be submitted to Phys. Rev. D (1993).

[5] A. M. Abrahams and C. R. Evans, submitted to Phys. Rev. D (1993).

[6] D. M. Eardley, in Texas/PASCOS 92: Relativistic Astrophysics and Particle Cosmology, eds. C. W. Akerlof and M. A. Srednicki (N. Y. Acad. Sci., New York), 1993.

[7] D. M. Eardley, Commun. Math. Phys. 37, 287 (1974).

[8] We use natural units in which $G=c=1$.

[9] L. I. Sedov, Similarity and Dimensional Methods in Mechanics, 10th Edition (CRC Press: Boca Raton), 1993. The self-similar solution described here may be equivalent to the 'exploding' models of Ref. [11].

[10] G. Guderley, Luftfahrtforschung 19, 302 (1942). See also the discussion of this problem in G. B. Whitham, Linear and Nonlinear Waves, (Wiley: New York), 1974. A spherically-symmetric imploding shock wave asymptotically approaches a self-similar form and "remembers" about the initial conditions only a single number related to the strength of the initiation of the shock.

[11] A. Ori and T. Piran, Phys. Rev. D 42, 1068 (1990).

[12] D. M. Eardley, talk at Numerical Astrophysics: A Symposium in Honor of James R. Wilson, at the University of Illinois, 1982 (unpublished); See e.g., J. M. Bardeen and T. Piran, Phys. Rep. 96, 205 (1983); L. I. Petrich, S. L. Shapiro and S. A. Teukolsky, Phys. Rev. D 33, 2100 (1986); R. F. Stark and T. Piran, Phys. Rev. Lett. 55, 891 (1985). 
[13] G. I. Barenblatt and Ya. B. Zel'dovich, in Ann. Rev. Fluid Mech., 4, 285 (1972). See their discussion of the modified blast wave problem on p. 287. 


\section{FIGURES}

FIG. 1. Self-similar radiation fluid collapse. Fluid and metric variables are plotted versus self-similarity coordinate $\xi=r / C(-t)^{n}$ (with $\left.n \simeq 1.1485\right)$ : (1) radial metric function $a(\xi)$ (solid), (2) velocity $V(\xi)=a U^{r} / \alpha U^{t}$ (dotted), (3) $\Omega(\xi)=4 \pi r^{2} \rho$ (dashed), and (4) lapse function $\alpha=n r N(\xi) / t$ (dot-dashed). Note the collapsing interior and expanding exterior regions.

FIG. 2. The mass function $m(r)$ (solid) at a late time in a supercritical evolution. Polar time slices limit outside the horizon producing the kink near where the function approaches the curve (dotted) $r=2 m(r)$, locating the surface of the black hole and determining the hole's mass. At late times, the fluid bifurcates and forms an evacuated region immediately beyond the hole, indicated by the decrease of $\Omega=4 \pi r^{2} \rho$ (dashed) outside the hole. At larger radii, escaping fluid expands outward.

FIG. 3. Critical behavior of black hole mass. For $\eta>\eta_{c}, M_{\mathrm{BH}}$ is determined and fit to a power law of the form (8). Here log of $M_{\mathrm{BH}}$ is plotted versus $\log$ of the critical separation $\eta-\eta_{c}$. The power-law fit is indicated by the solid line and is determined by $\eta_{c}=1.018828234, K=3.27$, and $\beta=0.36$. We obtain black holes with masses down to $M_{\mathrm{BH}} \simeq 0.001 M_{\mathrm{ADM}}$ and the scaling law (8) is seen to hold over two orders of magnitude in mass. Simulations with discretization scales of $3.3 \times 10^{-3}$ and $1.0 \times 10^{-3}$ are included in the plot, using open and filled circles, respectively.

FIG. 4. Intermediate asymptotic approach of a near-critical evolution to self-similarity. The metric function $a(r)$ (light curves) at a series of times, equally spaced in logarithm of central proper time $\tau=\log (-T)$, obtained from a near-critical evolution. The rightmost curve is near the beginning of the simulation and resembles $a(r)$ in the Cauchy data. As collapse proceeds, the peak of $a(r)$ shifts to progressively smaller scales. The metric and fluid approach the self-similar solution depicted in Fig. 1. The self-similar form of $a(\xi)$ (see Fig. 1) is overlaid at $\tau=-0.5$, clearly indicating approach to self-similarity. At later times, self-similarity is broken as a black hole begins to form and $a \rightarrow \infty$ on the horizon. 
This figure "fig1-1.png" is available in "png" format from: http://arxiv.org/ps/gr-qc/9402041v1 


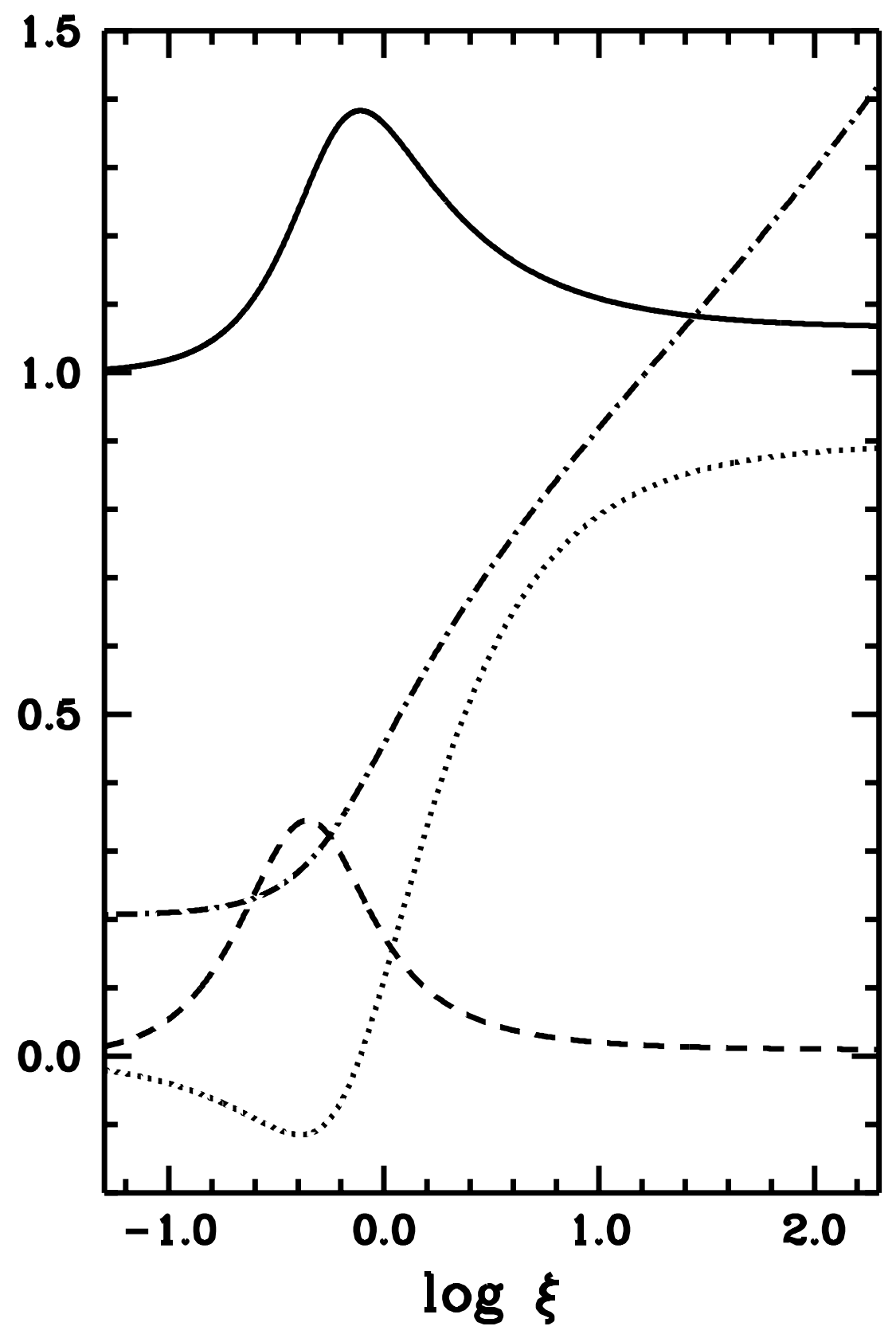

Figure 1 
This figure "fig1-2.png" is available in "png" format from: http://arxiv.org/ps/gr-qc/9402041v1 


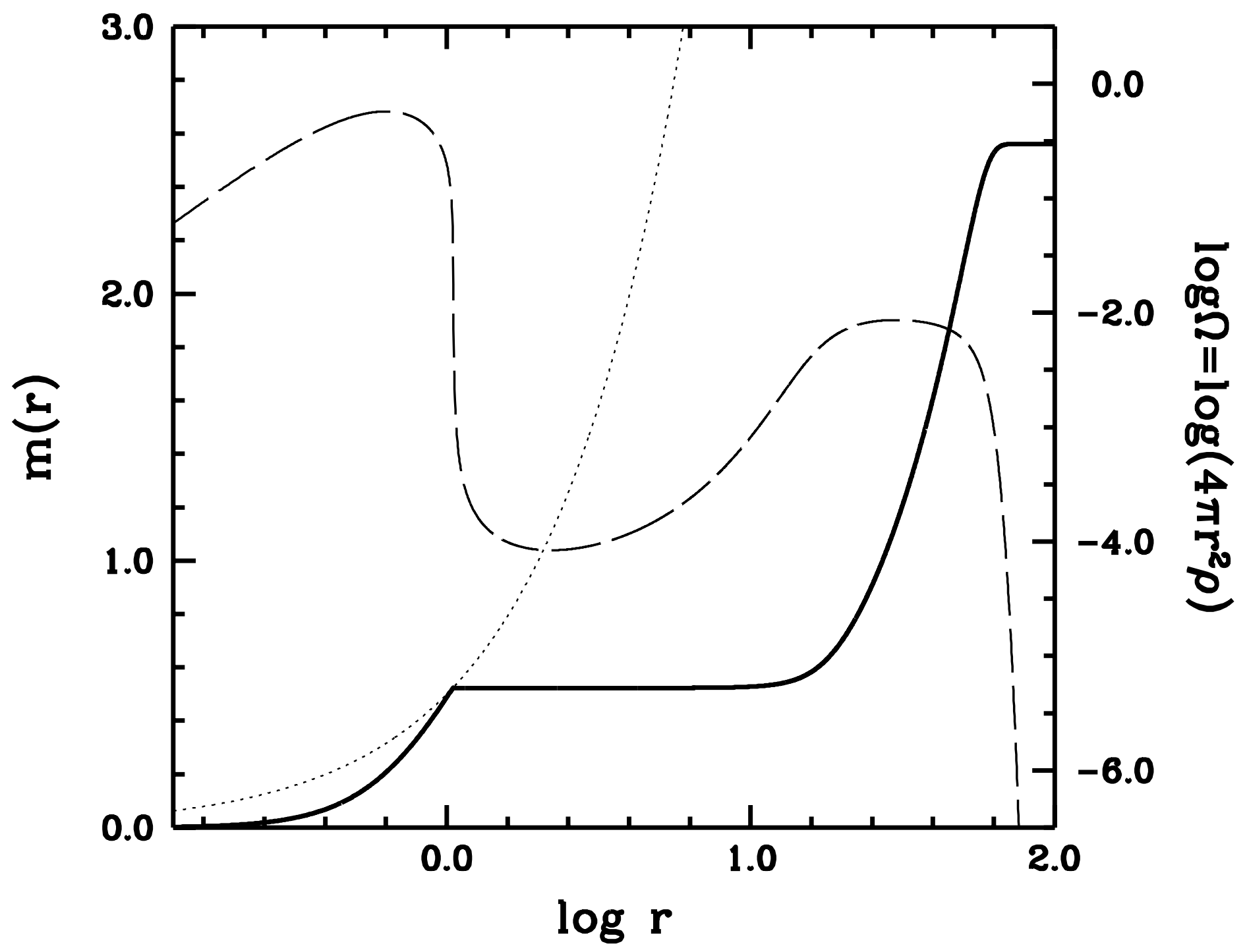

Figure 2 
This figure "fig1-3.png" is available in "png" format from: http://arxiv.org/ps/gr-qc/9402041v1 


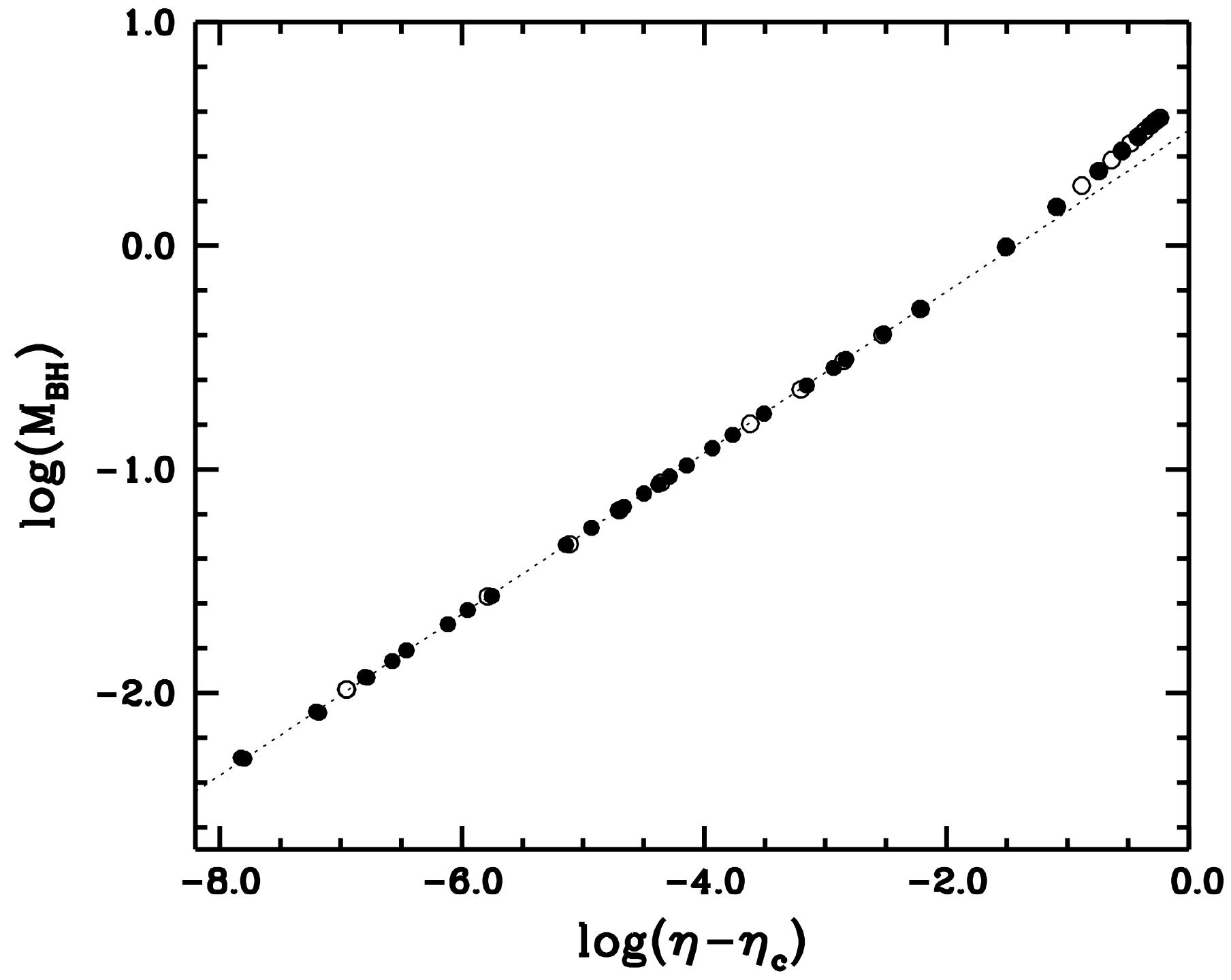

Figure 3 
This figure "fig1-4.png" is available in "png" format from: http://arxiv.org/ps/gr-qc/9402041v1 


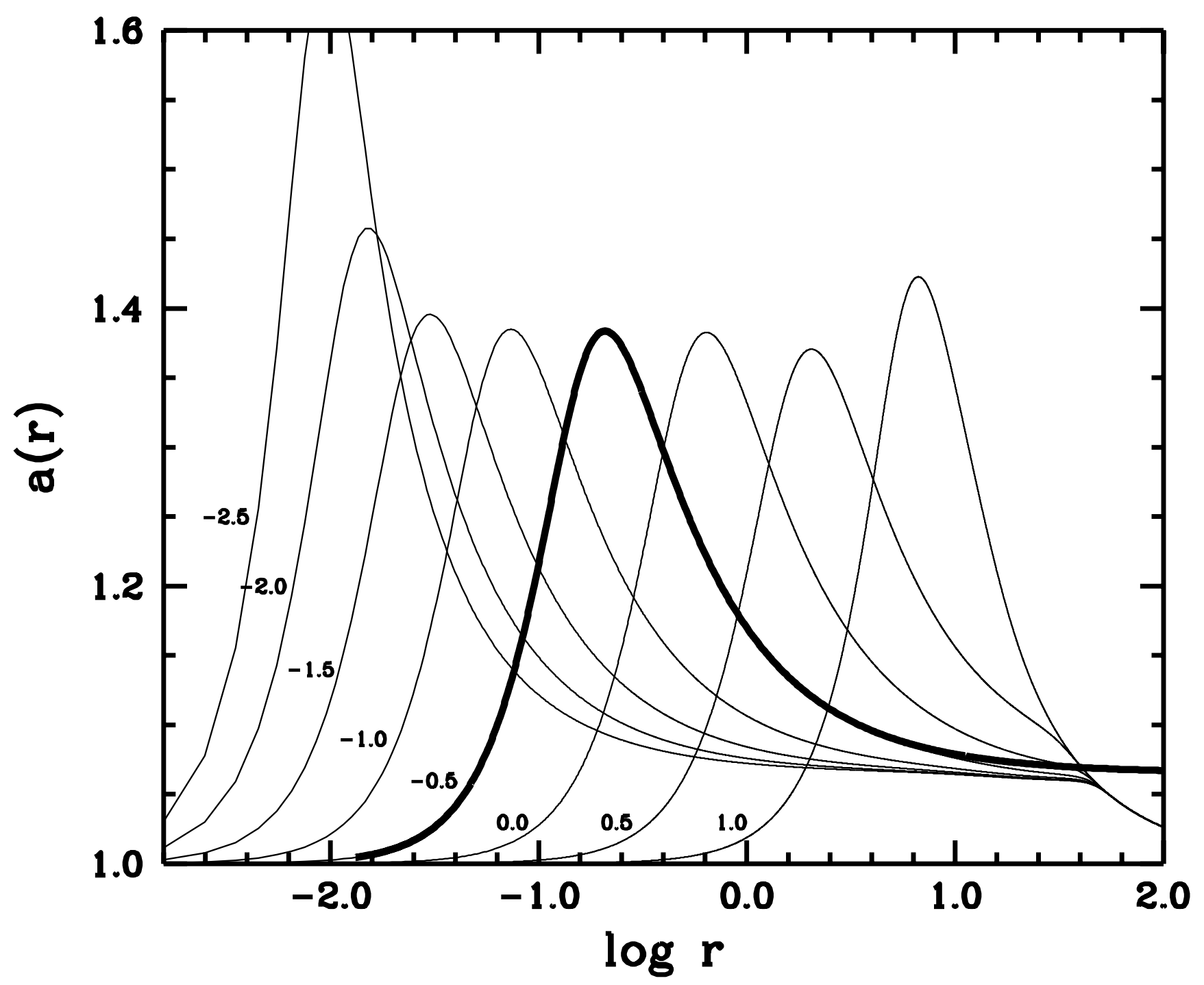

Figure 4 